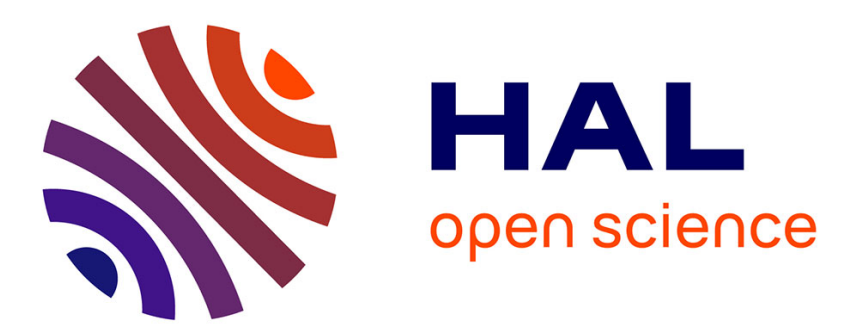

\title{
Influence of kinetic roughening on the epitaxial growth of silicon
}

\author{
Joël Chevrier, A. Cruz, N. Pinto, Isabelle Berbezier, J. Derrien
}

\section{To cite this version:}

Joël Chevrier, A. Cruz, N. Pinto, Isabelle Berbezier, J. Derrien. Influence of kinetic roughening on the epitaxial growth of silicon. Journal de Physique I, 1994, 4 (9), pp.1309-1324. 10.1051/jp1:1994190 . jpa-00246993

\section{HAL Id: jpa-00246993 https://hal.science/jpa-00246993}

Submitted on 1 Jan 1994

HAL is a multi-disciplinary open access archive for the deposit and dissemination of scientific research documents, whether they are published or not. The documents may come from teaching and research institutions in France or abroad, or from public or private research centers.
L'archive ouverte pluridisciplinaire HAL, est destinée au dépôt et à la diffusion de documents scientifiques de niveau recherche, publiés ou non, émanant des établissements d'enseignement et de recherche français ou étrangers, des laboratoires publics ou privés. 
Classification

Physics Abstracts

$68.55-61.14 \mathrm{H}$

\title{
Influence of kinetic roughening on the epitaxial growth of silicon
}

\author{
J. Chevrier, A. Cruz, N. Pinto $\left({ }^{*}\right)$, I. Berbezier and J. Derrien \\ CRMC2 CNRS, Campus de Lumıny, case 913, 13288 Marseille Cedex 09, France
}

(Receved 11 February 1994, lerised 17 May 1994, accepted 7 June 1994)

\begin{abstract}
The low temperature homoepitaxial growth of silicon has been probed in situ by Reflection High Energy Electron Diffraction (RHEED) in a Molecular Beam Epitaxy (MBE) chamber in the temperature range $250{ }^{\circ} \mathrm{C}-400{ }^{\circ} \mathrm{C}$. The continuous change in the RHEED patterns during the growth of thick films (several hundred angstroms) shows the progressive appearance of a surface roughness durng and after the decay of RHEED oscillations. This is a clear evidence for kinetic roughening in the case of silicon epitaxial growth at low temperatures on the Si(111) face. The surface width, $\sigma$, measured as the film thickness, $h$, is increased, can be described by: $\sigma(h, T) \sim A h \exp \left(E_{\mathrm{f}} / k T\right)$ with $E_{\mathrm{f}} \sim 0.65 \mathrm{eV}$. This is in marked difference with the kinetic roughenıng behavior measured during the growth of a metal like iron by means of the same RHEED technique (see Chevrier et al., Europhys. Lett. 8 (1991) 737). Furthermore, an extrapolation of this behavior to epitaxial growths at higher temperatures $\left(T \sim 600{ }^{\circ} \mathrm{C}-800{ }^{\circ} \mathrm{C}\right)$ suggests an effective influence of kinetic roughening in the determination of growth temperatures generally used for silicon MBE (i.e. the empirical usual growth temperatures $T_{\mathrm{ep}} \sim 650^{\circ} \mathrm{C}$ ). During growth at substrate temperatures between $250{ }^{\circ} \mathrm{C}$ and $400{ }^{\circ} \mathrm{C}$, the nucleation of misoriented silicon islands takes place following the occurrence of kinetic roughening at the surface. In this range of temperatures, this suggests that the loss of epitaxy occurs on a rough surface through the proliferation of defects and of misoriented crystals.
\end{abstract}

\section{Introduction.}

In the field of silicon-MBE crystal growth, a large development of techniques has enabled one to grow high quality silicon crystals in ultra high vacuum conditions. Hence the growth of quasi-perfect homoepitaxial thin films is currently achieved in many laboratories. Nowadays, the number of studies produced in this field just prevent any attempt to collect a comprehensive bibliography. The major stakes and industrial applications are extensively describes in reference [1] Looking at the huge number of experımental results, one may think that the most

(*) Present address : Universita Deglı Studi di Camerıno, Dipartımento di Matematıca e Fisıca, Via Madonna delle Carcerı, 62032 Camerıno, Italy 
important physical mechanisms involved in silıcon-MBE are well known. Indeed the key experimental conditions to grow high quality silicon crystals are very well identified. However, there still exist major questions, with, to our knowledge, only limited answers.

For example, a crystal to amorphous transformation has been well identified during the MBE growth of silicon at low temperatures. This is a major feature of the silicon growth which prevents the growth of thick epitaxial films at low temperatures. However the mechanisms which spontaneously drive a single crystal of silicon into a completely disordered amorphous state during the growth at low temperatures, are still a matter of debate [2] and this is certainly an important issue in the understanding of epitaxial growth as a non-equilibrium process.

Furthermore, in order to establish the optimal experimental conditions for the MBE epitaxial growth of silicon, many attempts have been made to define a minimum temperature of the silicon crystal during silicon atom deposition [3]. The fact that MBE growth is a nonequilibrium process has only recently been fully included in the experimental analysis. This approach states that the continuous change of the crystal surface during the complete growth process must be taken into account to describe the final crystalline state [2, 4, 5]. A consequence of this point may be that the definition of the minımum temperature for the silicon epitaxial growth should be replaced by the determination of the thickest flat epitaxial film that one can grow at a given temperature. Such a statement is experimentally supported by the fact that at very low temperatures (i.e. around room temperature), the occurrence of the epitaxial growth has been clearly demonstrated [6]. The first stages of the silicon growth at low temperatures have definitely been shown to be of a reasonable epitaxial quality by transmission electron microscopy [2] before the epitaxial growth finally deteriorates as the thickness is increased. This shows that, at least in the low temperature regime, a continuous and irreversible transformation of the growing film structure is observed. The growth does not present a strick layer by layer growth mode and is not exactly periodic in times. This is only at higher temperatures (in the vicinity of the usual growth temperature), in good experimental conditions of growth, that no clear deterioration of the crystalline quality is observed throughout the whole film. In such a case, the electron reflectivity measured during the growths shows large RHEED oscillations with almost no noticeable damping even after deposition of several hundred silicon layers [7]. Incidentally, one may notice that if the observation of RHEED oscillations is a necessary step in the experimental control of MBE growth of metals or semiconductors, a detailed understanding of the surface characteristics controlling their amplitude and their damping only recently emerged [4]. Quite clearly real space techniques such as Scanning Tunneling Microscopy, Reflection Electron Microscopy and Low Energy Electron Microscopy have been at the heart of theses improvements. Together with Field Emission Microscopy [8], they have provided new results on the microscopic mechanisms of atomic transport on surfaces $[4,9,6]$.

In this framework, the aim of this article is to experimentally describe the contunuous changes of the RHEED patterns observed in situ durıng epitaxial growth of thick silicon films $(\sim 500 \AA)$ at different temperatures $\left(250^{\circ} \mathrm{C}<T<400^{\circ} \mathrm{C}\right)$. The interpretation of the diffraction peak shape will enable us to conclude that a kinetic roughness appears during growth and produces a surface in a non-equilibrium state. Together with the analysis of RHEED patterns, the ability to vary the growth temperature offers the possibility to investıgate the development of this kinetic roughenıng and the loss of epitaxial growth in different conditions of atomic transport on the surface. This will also provide us with a quantitative surface description of how the epitaxial growth improves as the growth temperature is raised. Our complete results give some further insight into the reason why the definition of an epitaxial temperature in the field of silicon MBE was quite natural but also somewhat obscure as illustrated by a dispersion of about $200^{\circ} \mathrm{C}$ in its definition for the (111) silicon surface [3]. 
Furthermore theoretical analyses have described how random deposition of atoms on surfaces triggers the development of this kinetic roughening during epitaxial growth [10-12]. An important motivation for the theoretical studies of the surface morphologies during epitaxial growth of thick films is the apparent lack of characteristic time and length in the growth dynamics. This is thought to lead a characteristic parameter like the surface width to vary as a power law of time. In a previous experimental investigation using the same RHEED technique, we have experimentally observed the occurrence of a kinetic roughening at the surface during the epitaxial growth of iron at low temperatures [5]. In this case, following the same experimental procedure as described in the next section of this article, the analysis of the Bragg peak shape in the RHEED pattern led us to characterize the continuous development of the kinetic roughness as the film thickness was increased. Directly deduced from the length of RHEED peaks, the roughness perpendicular to the surface, $\sigma$, was found to be linked with the film thickness, $h$, by the following relationship :

$$
\sigma \propto h^{\beta}
$$

with $\beta \sim 0.25$. This power law is consistent with the expected scale invariance of the surface morphology during the development of kinetıc roughening. Furthermore the exponent $\beta=0.25$ is close to the value which is theoreticaly predicted for a kinetic roughening driven by the random deposition of atoms and relaxed by surface diffusion mechanisms. These results have been experimentally confirmed and completed by a recent study of iron epitaxial growth by means of in situ High Resolution Low Energy Electron Diffraction [13]. A behavior comparable to the one described by equation (1) has been found with an exponent $\beta \sim 0.22+/-0.022$. This is indeed quite consistent with our results although both studies have not considered the same iron crystalline face.

The experimental analysis of surface roughness occurring during the homoepitaxial growth of silicon on the silicon (100) silicon at low temperatures has revealed the occurrence of a similar kinetic roughening [14]. Analysis of this roughness has shown that the surface width can be reasonably described using equation (1). However the most consistent exponent with experimental results is $\beta \sim 1$. As pointed out in many references, it is reasonable to think that $\beta$ should always be smaller than 0.5 if the surface diffusion relaxes the occurring roughness [10]. Therefore such an experimental finding basically implies that the roughness introduced by random deposition of atoms could be amplified by the mass transport mechanisms on the surface. This could find its origin in the experimental results on surface diffusion mechanısms deduced from Field Emission Microscopy [8]. A key point of these findings is the existence of a potential barrier at the upper part of the terrace edge. Whenever this barrier is present, it can lead during atomic deposition to an effective lack of mass transport between terraces of different heights. During the first stages of epitaxial growth, the dramatic influence of such mechanism on the occurrence of terraces with various heights has been demonstrated [4]. A numerical simulation presented in reference [14] has tested the efficiency of this restricted diffusion in developing surface roughness durıng epitaxial growth of thick films. The results suggest that a lack of atomic exchange between terraces of different heights causes a surface roughness during epitaxial growth which is much more important than that found when no barrier is taken into account.

Therefore it is a motivation of this article to investigate, in the same experimental conditions, the surface morphology observed during growth for two very different systems such as iron and silicon. This should enable us to provide some new insights into the kinetic roughening of thick growing films [13, 14]. 


\section{Experimental.}

Silicon growth on (111) oriented silicon wafers (two inch diameter) was performed in an MBE machine equipped with a silicon evaporation cell and a $10 \mathrm{kV}$ electron gun for RHEED experiments. The base pressure of the MBE chamber at room temperature was between $1 \times 10^{-10}$ and $2 \times 10^{-10}$ torr. The silicon source consisted of an evaporaton cell using a thermally heated graphite crucible. The temperature control was better than $1{ }^{\circ} \mathrm{C}$ for both the substrate and the evaporation cell as it was measured on their own thermocouple. The accuracy of the temperature measurement was controlled on the crystallization of amorphous silicon and on the $7 \times 7 \leftrightarrow 1 \times 1$ transition at the sllicon surface : the temperature shift should not be larger than about $20^{\circ} \mathrm{C}$. Durıng silicon evaporation, the cell temperature was kept at $1360^{\circ} \mathrm{C}$. Cleaning of the initıal silıcon surface was based on two stages : i) an ex situ chemıcal etching based on the classical Shiraki procedure, ii) a desorption of the native oxide by heating the wafer at $800^{\circ} \mathrm{C}$ associated with an atomic beam of silicon directed onto the surface. Such a treatment ended up in a reproducible, clear $7 \times 7$ reconstructed silicon surface. For this particular study of silicon growth at low temperatures several growths could be achieved on the same sample with a good reproducibility of RHEED results provided that a long annealıng at $800{ }^{\circ} \mathrm{C}$ was performed followed by the growth of a buffer of roughly 50 monolayers at about $800^{\circ} \mathrm{C}$. After this treatment, the RHEED pattern was very close to the initial one. However such a procedure could not be used when the signature in the RHEED pattern of nucleation of new and misoriented crystals did appear. Even after a long annealing at high temperatures, it is hardly possible to recover the usual RHEED pattern of silicon in the case. We shall also see that repeated cycles of growth and annealing ended up in an increase of the residual roughness. The influence on the developing roughness during the growth of these changing initial conditions will be analysed in the next section.

Our electron diffractometer is a conventional instrument comparable to many systems used to characterize epitaxial growth. The measurements presented here have been made possible owing to a systematic video acquisition of diffraction patterns. The camera we have used to record the RHEED patterns on the phosphorescent screen has a sensibility of 0.3 lux. Digitalisation of the video signal is performed through an 8-bit computer card (Optiscan from Neotech). which means that the dynamic of this detector is limited to 256 grey levels. During the measurements, great care has been taken to avoid saturation either on the screen or during picture acquisition. The grazing angle of the electron beam with the surface was about one degree. The camera periodically took pictures of the RHEED patterns (the acquisition period could be changed from 1 picture/s up to 1 picture/day) which were stored in the computer. Image analysis was then performed. Both intensities of diffraction spots and their widths (perpendicular and parallel to the sample surface) are numerically extracted. The complete digitalisation of diffraction patterns enabled us to analyse the position and the shape of the Bragg peaks.

During growth, measurements of RHEED oscillations have been performed by a selective record of the reflected specular beam. The periodic changes of intensity of this small area in the RHEED screen were clearly visible to the naked eye. One may notice that such a large variation was not observed in another part of the screen. Compared to the average reflectivity during growth, the variation of intensity during oscillations could be as large as $25 \%$ without any correction for the background. In this article, we are not going to experimentally provide new insights into the growth mechanisms which determine the oscillations of electron reflectivity. It seems to us that it would be quite difficult to obtain new significant results on the sole basıs of electron diffraction at grazıng incidence. An experımental basis of this remark is the observation that the damping of RHEED oscillations measured during growth varies from sample to sample with different initial surface treatments although the initial RHEED patterns 
are hardly different. Also in the case of our experimental set up, i.e. a non-rotating sample with a diameter of two inches, even a small non-homogeneity of the sılicon beam would induce an apparent damping of the RHEED oscillations. In our case, this last point almost completely prevents any attempt to analyse the damping of RHEED oscillations quantitatively. Motivation for the measurement of these oscillations is twofold. First, this enabled us to determine the silicon flux. Figure 1 reports the RHEED oscillations measured during the silicon growth on a (111) silicon surface with a substrate temperature of $T \sim 350^{\circ} \mathrm{C}$. Analysis of 10 periods leads to a silicon flux of about 1 bilayer per minute (more precisely an increase of thickness $a_{0} / \sqrt{3}=3.13 \AA$ every $65 \mathrm{~s}$ ). Also the observation of large amplitude RHEED oscillations during growth is a good indication that the cleaning procedure, the sample handling and the growth conditions are reasonable.

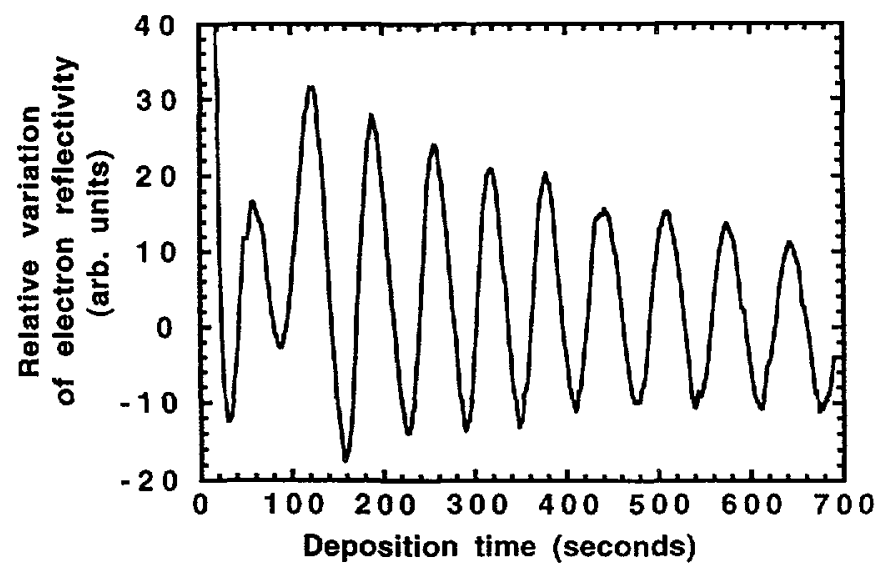

Fig. 1. - RHEED oscillations observed by recording intensity of the specular beam during silicon growth at $T=350^{\circ} \mathrm{C}$ on (111) silicon surface.

Beside RHEED oscillations, a second important effect is visible to the naked eye during silicon growth. Starting with the typical RHEED pattern of a flat ordered silicon surface, the condensation of RHEED streaks into conventional Bragg spots of bulk silicon occurs. In this case, the positions of Bragg peaks can be analysed in a way very much comparable to what is done for diffraction patterns produced by Transmission Electron Microscopy. This continuous change from a typical two-dimensıonal diffraction pattern to a three-dimensional pattern is a clear evidence for the occurrence of surface roughness. Indeed in the reciprocal space, the length of reciprocal rods perpendicular to a flat initial surface is primarily determined by the short penetration depth of the electron beam. As the surface becomes rough, the electron beam goes through surface bumps and the reciprocal rods condense towards bulk diffraction peaks (An illustrated description of these mechanisms can be found in ref. [15]). In the direction of the reciprocal space, perpendicular to the surface, the surface roughness imposes a limited length to the diffraction peaks. The general fact that the width of the diffracted peak is inversely proportional to the size of the diffracting object provides a direct relationship between the perpendicular Full Width at Half Maximum of the diffraction peaks, $\Delta G_{\perp}$, and the surface width, $\sigma$, which is characteristic of the surface roughness and corresponds to the effective sample size experienced by the electron beam :

$$
\Delta G_{\perp}=2 \pi C / \sigma
$$


where $C$ is a calibration constant that is determined using the distance between the $1 \times 1$ silicon streaks. The use of this quantitative relationship relies on restrictive approximations. A description of the RHEED technique with its mains limitations can be found in reference [15]. In the particular case of silicon growth at low temperatures, limitations due to two severe approximations are directly observed in the RHEED patterns. A first approximation is that the measured intensity along a RHEED streak is primarily determined by the surface morphology and not by an instrumental effect. This especially supposes that the intersection of the reciprocal space with the Ewald sphere has essentially no influence on the value of $\Delta G_{\perp}$. In the case of a clean and well ordered silicon surface before growth, this is certainly far from to be correct. This has been shown in detail in [15]. For a very flat and ordered surface, the lengths of RHEED streaks are essentially determined by the intersection of the Ewald sphere and the weakly modulated reciprocal rods. As seen in figure 2, this intersection is the origin of large circles formed by short RHEED streaks in silicon diffraction patterns (see also [16]). In the case of very flat and ordered surfaces observed with a narrow beam of very small divergence, this effect can be so large that one observes circular spots. For the results presented here, this has two main consequences: 1 ) it is quite difficult to quantitatively estimate the initial roughness on the surface, ii) one has to make sure that durıng the silicon growth, the apparent shape of RHEED streaks is dominated by the sample itself and not by the intersection with the Ewald sphere. During deposition of the first few layers deposited at growth temperatures around $T \sim 300{ }^{\circ} \mathrm{C}$, the diffraction pattern is deeply changed. The RHEED streaks due to the $7 \times 7$ reconstruction are quickly weakened and those characteristic of the $1 \times 1$ silicon structure are laterally broadened. A consequence of this is a longer intersection of diffraction streaks with the Ewald sphere resulting in long streaks in the RHEED patterns. This is qualitatively the RHEED pattern expected from a flat surface with a deteriorated crystalline order. The length of these RHEED streaks at the beginning of the growth shows the maximum intersection between the modulated reciprocal rod space and the

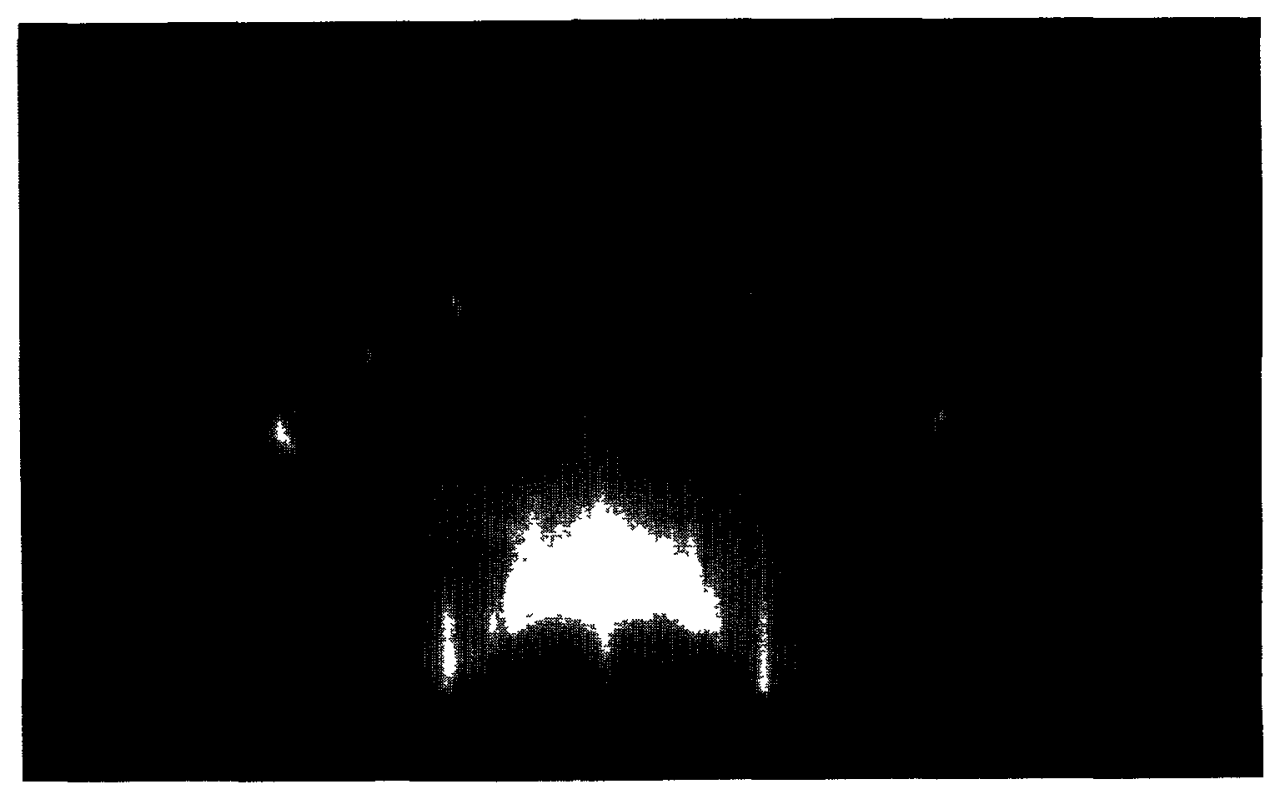

Fig. 2. - RHEED pattern observed on a clean (111) silicon surface before growth with electron beam along [1T0] azımuth. 
Ewald sphere. This indicates that the roughness measurement is reasonable as long as the analysis is confined to Bragg spots close to the center of the reciprocal space. The results presented here have all been acquired under those conditions. The Bragg spots generally used for our experiments are those with Miller indices 113 and 220 (the electron beam is along a [1 10$]$ azimuth and the axis perpendicular to the surface is the $\langle 111\rangle)$. A further consequence of these experımental limitations is the range of growth temperatures that we can use for a study of kinetic roughening by means of RHEED. Indeed above a growth temperature of $T \sim 400^{\circ} \mathrm{C}$, evidence for the appearance of roughness in the RHEED patterns during the epitaxial growth is limited. This prevents a quantitative analysis of the roughness based on grazing incidence electron diffraction.

A second approximation is that the variation of $\Delta G_{\perp}$ can be analysed as the quantitative signature of only one mechanism : the occurrence of the surface roughness. In the case of the growth of a perfect crystal, the variation of $\Delta G_{\perp}$ in the reciprocal space would be directly connected to the occurrence of a surface roughness. In our RHEED patterns, a characterization of the crystalline quality lies in the width of Bragg peaks parallel to the surface as there is no relaxation of the Bragg condition in this direction due to the limited penetration of electron. In the temperature range we have investigated, a lateral broadening of the Bragg peaks is observed which shows that the crystalline quality deteriorates during the growth. This certainly means an equivalent broadening in all directions of the Bragg peaks. As the strong reduction of Bragg peak length, $\Delta G_{\perp}$, cannot be due to the crystal disorder, it clearly remains that the decrease of $\Delta G_{\perp}$ is strongly associated with the occurrence of a surface roughness. Quantitatively it may appear difficult to separate the effect of roughness which tends to shorten the Bragg peaks and the appearance of a poor crystalline quality which gives rise to a broadening of Bragg peaks. Indeed in the final stage of the growth, the Bragg peaks are nearly circular. In this case, it would be extremely difficult to safely separate the contribution of surface roughness and of atomic disorder from the peak width. This is only when one contribution is dominant that it is possible to analyse the streak shape quantitatively. The criterion we have used is that the streak length perpendicular to the surface must be much longer than the peak width parallel to the surface which is primarily determined by the cristalline quality $[17,5]$. Therefore the two extreme regimes described here impose constraints on the use of $\Delta G_{\perp}$ as a quantitatıve signature of the surface width.

\section{Results.}

3. 1 GENERAL OBSERVATIONS. - In figure 3, pictures of the full RHEED screen are presented at different stages of the growth for a substrate temperature at $T=280^{\circ} \mathrm{C}$. The electron beam is along a $[1 \overline{1} 0]$ silicon azimuth. Signatures of the major effects which are quantitatively investigated in this article are clearly seen in this figure 3. Compared to figure 1, p1cture a of figure 3 taken after deposition of a few layers on the surface shows the disappearance of the $7 \times 7$ diffraction streaks with long and rather broad $1 \times 1$ streaks. Such a change of the diffraction pattern is consistent with a limited roughness and disorder within the surface. Further deposition (picture b of Fig. 3) leads to a complete disappearance of the $7 \times 7$ reconstruction and to a condensation of Bragg spots. Such an effect is seen for all spots of the diffraction pattern. This is a strong evidence for the continuous occurrence of a surface roughness during the growth. Qualitatively such a result is very much comparable to what is observed in other cases $[5,6]$. In this range of temperatures further sulicon growth clearly induces new spots in the diffraction pattern (picture c of Fig. 3). These new spots call for several remarks : 1) these are new, localised and well defined spots. No diffraction rings are observed at the same time in the diffraction pattern. 11) These new spots do not appear at the 


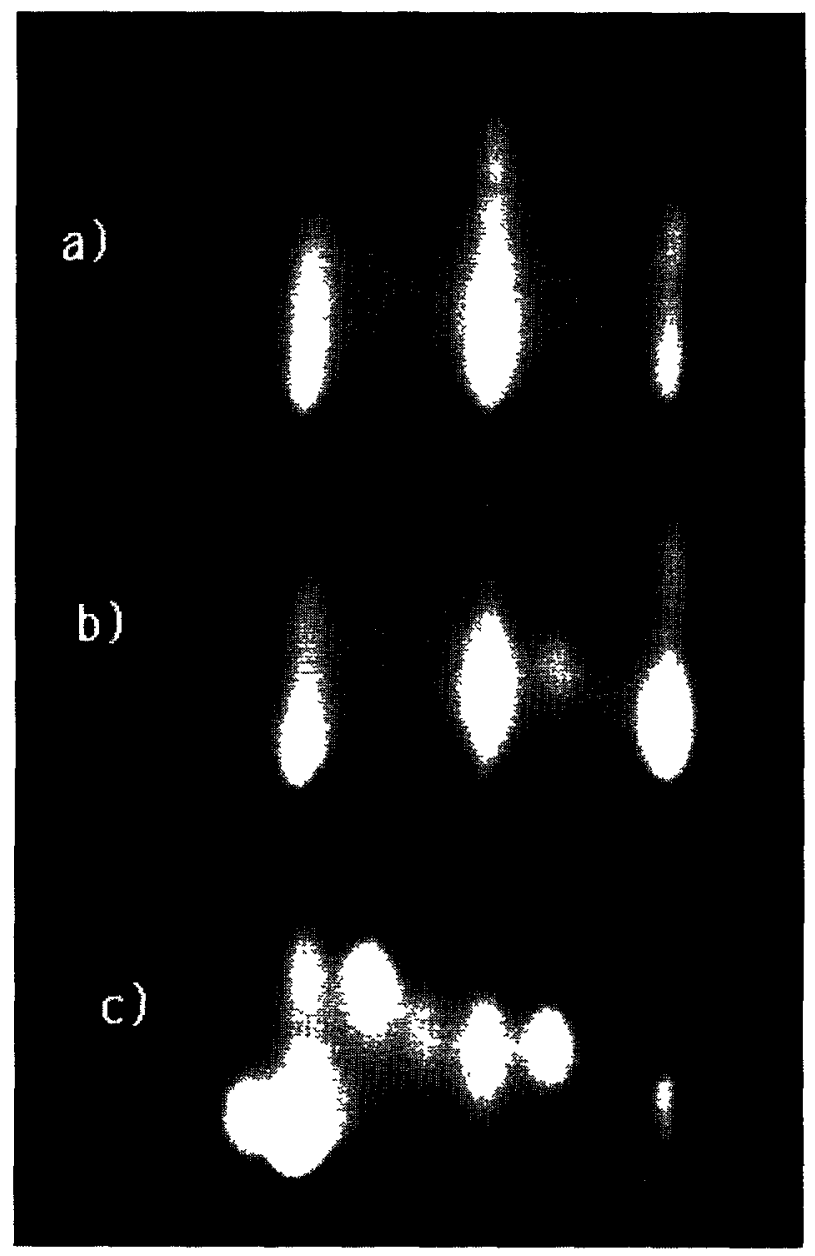

Fig. 3. - Characteristic diffraction patterns observed during depositıon of sllıcon at $T=280^{\circ} \mathrm{C}$.

very beginning of the growth but after development of some roughness on the surface. iil) This is a highly reproducible result in this range of temperatures. However, for growth temperatures above $400^{\circ} \mathrm{C}$ and for the thicknesses investigated, the occurrence of these new Bragg spots is hardly seen. This observation will be more quantıtatıvely developed in a following paragraph with the determination of the crystallographic orientation of these new crystals on the substrate. Already, this is an evidence for the nucleation of new crystallites on the surface and for an original mechanısm leading to the loss of epitaxial growth during silicon deposition on silicon (111) surface in the temperature range $250{ }^{\circ} \mathrm{C}-400{ }^{\circ} \mathrm{C}$. At lower temperatures such a behavior is not clearly observed: the whole original diffaction pattern disappears after condensation of streaks into Bragg spots. This means that the complete disappearance of the diffraction pattern follows the occurrence of a rough surface. After this 1rreversible transformation, only large and diffuse rings are left on the screen. This is the commonly reported evidence for the RHEED observation of amorphous silicon. An important experimental observation already reported at other growth temperatures $[6,7]$ is that, after the atomic flux is stopped, no change of the silicon surface is observed at the growth temperature (always 
below $400{ }^{\circ} \mathrm{C}$ ). However heating the sample at a higher temperature (around $700{ }^{\circ} \mathrm{C}$ ) induces an irreversible flattening of the surface and the diffraction evidence for the $7 \times 7$ silicon reconstruction is recovered.

These major and general observations of the kinetic roughening during the silicon growth at low temperatures are consistent with what we have previously reported for the growth of iron. This emphasizes how much general this effect is. The specific and quite important effect observed for silicon is the loss of the epitaxial growth of a single crystal. This is in marked difference with the epitaxıal growth of iron [5] for which no qualitatıve loss of the epitaxial growth was observed even though a comparable kinetic roughening was observed as seen by the RHEED technique.

3.2 QUANTITATIVE ANALYSIS OF KINETIC ROUGHENING. - The variation of the surface width, $\sigma=2 \pi C / \Delta G_{\perp}$, versus the film thickness is displayed in figure 4 for two different temperatures. The first observation concerns the absolute value of the roughness measured. For a total film thickness of about $500 \AA$, this roughness is in the nanometer range. This is quite comparable to what has been observed for the iron epitaxial growth. It is also very close to what is reported for the growth of silicon on silicon (100) surface in the equivalent temperature range [14]. These measurements have been performed in direct space by Transmission Electron Microscopy whereas our results have been obtained by means of a reciprocal space technique. A second point is that the importance of substrate temperature definitely appears in figure 4. At a growth temperature of $T=400{ }^{\circ} \mathrm{C}$, the occurrence of roughness is rather limited and this is even the limit we have been able to investigate by RHEED. At $T=300^{\circ} \mathrm{C}$, the change of the surface is much quicker and the kinetic roughening develops much more rapidly. Strictly looking at the experimental results in this range of film thicknesses, the best fit for the plot $\sigma$ versus $h$ is obtained at all temperatures with a linear dependence of the roughness with the film thickness. This is similar to the experimental result obtained during the growth on the (100) silicon surface [14]. This linear behavior is only approxımate but this is a clear difference with the behavior measured during the growth of iron [5].

Although the same kinetic roughening develops during epitaxial growth for two different systems like iron and sllicon, clear differences are experımentally observed in details using the same RHEED technique and the same analysis of RHEED patterns. These differences in the development of kinetic roughening are also quite consistent with other studies $[13,14]$ using

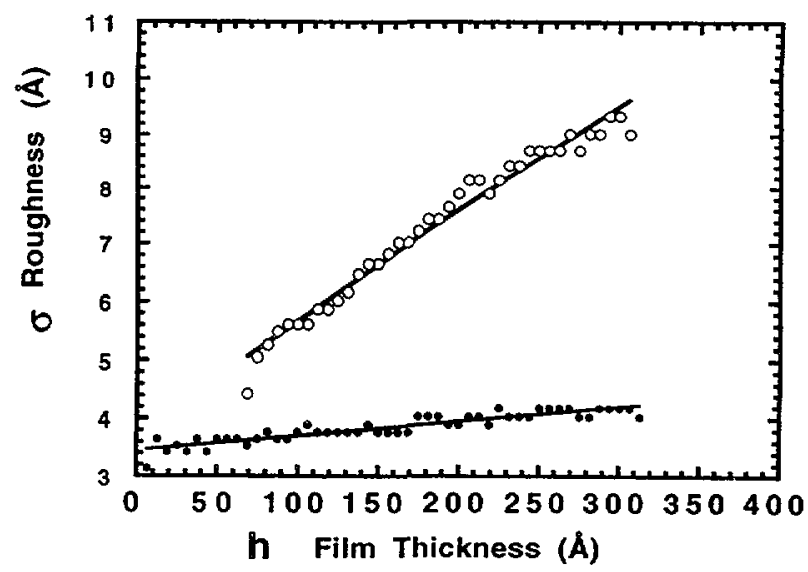

Fig. 4. - Roughness perpendicular to the surface deduced from the FWHM of diffraction Bragg peaks during the silicon deposition at $T=300^{\circ} \mathrm{C}(\mathrm{O})$ and at $T=400^{\circ} \mathrm{C}(\bullet)$. 
different techniques. Therefore all these experimental results emphasize the fact that the occurrence of kinetic roughening during low temperature epitaxial growth is a general effect, but they also suggest that a unique and simple analysis for all surfaces does not seem to exist. A description of the microscopic mechanisms responsible for this difference is beyond the grasp of our experimental results. However they directly lead to the evidence that, depending on the systems, the surface diffusion and nucleation can relax the surface morphology (this corresponds to an apparent exponent $\beta$ lower than 0.5 ) or amplify the surface roughness as shown by a measured exponent $\beta$ larger than 0.5 .

Durıng epitaxial growth at different temperatures, the analysis of the surface morphology can provide further insights into the influence of surface mass transport in the development of kinetic roughening. The curves $\sigma$ versus $h$ measured at different temperatures present the same shape. This means that we can reliably extract the slope $\mathrm{d} \sigma / \mathrm{d} h$ at different growth temperatures. This is essentially what is reported in figure 5. The plot $\log (\mathrm{d} \sigma / \mathrm{d} h)$ versus $1 / T$, the inverse of the growth temperature, strongly indicates that the general equation which describes the increasing roughness during growth is :

$$
\sigma=A \exp \left(E_{\mathrm{f}} / k T\right) h
$$

with $E_{\mathrm{f}} \sim 0.65+/-0.07 \mathrm{eV}$. Essentually this shows experimentally that, in the particular case of silicon, the appearance of the roughness during growth at low temperatures is controlled by a thermally activated process on the surface. The thermally activated processes on surfaces one can think of during epitaxial growth are basically the diffusion of silicon adatoms on a flat silicon surface and the nucleation of islands. The activation energy directly yielded by our analysis of roughness on the silicon (111) face is close to the measured activation energy $E_{\mathrm{a}} \sim 0.67 \mathrm{eV}$ reported in reference [9] for surface diffusion of adatoms on the (100) silicon face. Recent measurements have also reported an activation energy $E_{\mathrm{a}} \sim 0.7 \mathrm{eV}$ for surface diffusion of adatoms on the (111) silicon surface [18]. However, the identification of $E_{\mathrm{f}}$ with the activation energy $E_{\mathrm{d}}$ of adatom diffusion on silicon (111) surface cannot be reliably done at this point. Indeed an important experimental parameter in the control of the $\mathrm{MBE}$ growth process is the atomic flux on the surface. The influence of the silicon flux in the

Growth temperature

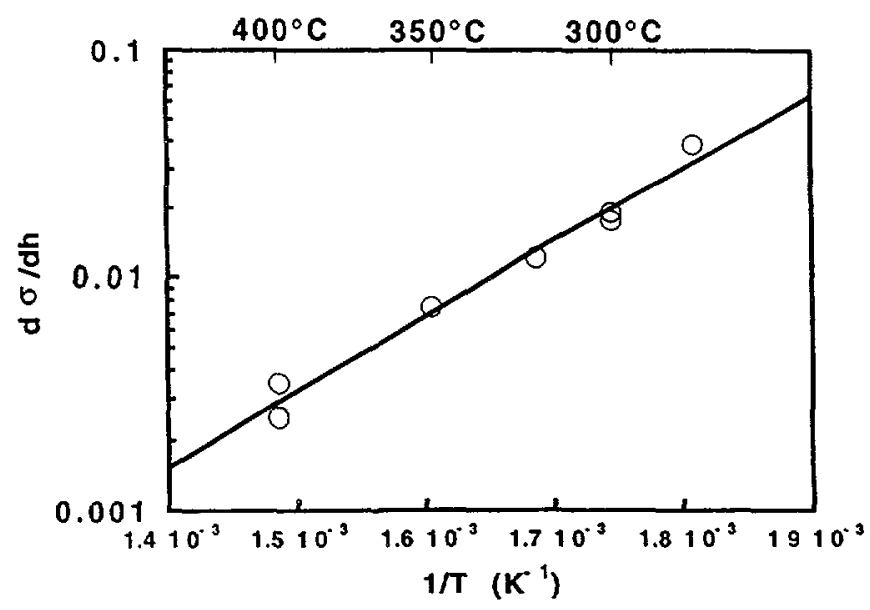

Fig. 5. - $\mathrm{d} \sigma / \mathrm{d} h$ versus $1 / T$. The straight line $1 \mathrm{~s}$ given by $\sigma=A \exp \left(E_{\mathrm{f}} / k T\right) h$ where $A \sim 4.6 \times 10^{-8}$ and $E_{\mathrm{f}} \sim 0.65 \mathrm{EV}$. 
development of surface roughness during growth is not described by our results. This means that we do not know whether or not $E_{\mathrm{f}}$ depends on the atomic flux. However a direct conclusion is that kinetic roughenıng is experımentally observed in a temperature regime where a large mobility of adatoms on a flat silicon surface clearly exists.

In order to link these experimental results with the common practice in MBE growth of silicon, we extrapolate equation (3) to a larger range of temperatures than the one we have measured. We take as a conventional and reasonable definition of a good quality silicon film grown by MBE, a roughness lower than $1 \AA$. Although such a low value is in fact not measurable by RHEED and would certainly correspond to a diffraction pattern hardly changed by the growth, this definition provides a reliable criterion. A slight change in the accepted final roughness of $1 \AA$ would not significantly alter this analysis. Figure 6 shows the maximum thickness one can grow with that constraint of low roughness at different growth temperatures. This curve clearly indicates that growth temperatures of about $600{ }^{\circ} \mathrm{C}-700{ }^{\circ} \mathrm{C}$ are needed to obtain flat surfaces after growth of thick silicon films (about $5000 \AA-10000 \AA$ ). It is clear that this plot is only an extrapolation of measurements performed at lower temperatures and that the film quality is not only determined by the surface flatness. Furthermore since we are dealing with kinetic effects induced by random deposition of atoms, one would expect these results to exhibit a dependence not only with temperature but also with the number of atoms coming on the surface. Using an evaporation cell, the sllicon flux we have used is between one or two orders of magnitude lower than the usual fluxes used in MBE equipped with electron guns. However, within our experimental conditions, the conclusion is that it is necessary to grow silicon at temperatures higher than $600{ }^{\circ} \mathrm{C}$ to remove significant effects of kinetic roughening in the growth of thick (111) silicon films. Despite the aforementioned limits of our experimental investigation, it is remarkable that this is what is currently done in silicon MBE growth. For example, in reference [3], epitaxial temperatures for (111) silicon are reported to be around $520^{\circ} \mathrm{C}$ and $700{ }^{\circ} \mathrm{C}$ depending on experimental characterizations used to define this epitaxial temperature. Also, as the film thickness in $\mathrm{MBE}$ growth required for applications must be in the range $1000 \AA-10000 \AA$, the curve in figure 6 can easily be approximated by a

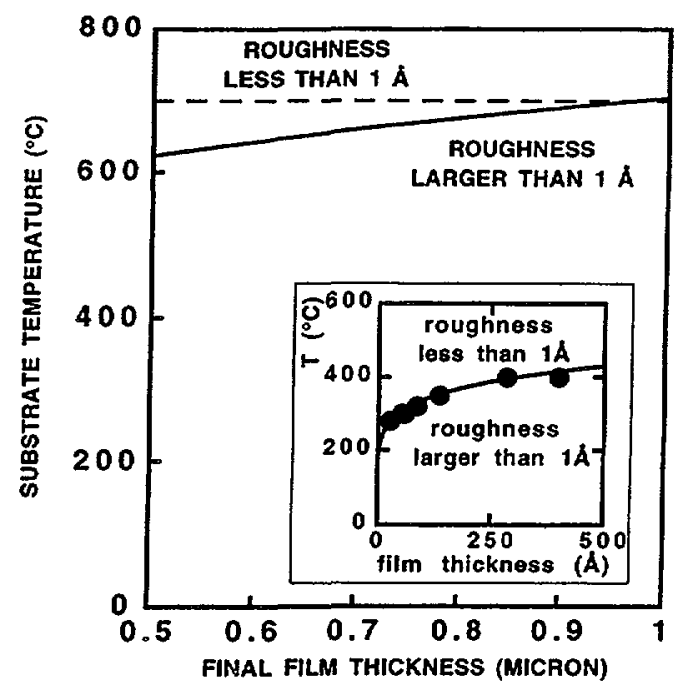

Fig. 6. - Estımate from figure 5 of the growth temperature range needed to grow an epitaxial silicon film with a thickness in the micron range and with a roughness lower than $1 \AA$. The inset with the experimental result shows the same curve for lower thicknesses and therefore lower growth temperatures. 
constant growth temperature of $700{ }^{\circ} \mathrm{C}$ for any desired thickness of a flat epitaxial silicon film. This is represented by the dotted line in figure 6 . This provides a plausible explanation for the determınation of an epitaxial temperatures and suggests that the kinetıc roughenıng occurrıng during silicon epitaxial growth is one of the parameters which implicity control the determination of conventional experimental conditions of silicon epitaxial growth.

In figure 7 , the development of roughness during epitaxial growth at $T=300{ }^{\circ} \mathrm{C}$ is presented for different inital surface morphologies. The main characteristic of these initial surfaces is that they have been obtained after several cycles of growth at low temperatures, annealing at high temperatures $\left(T \sim 800{ }^{\circ} \mathrm{C}\right.$ ) and growth of a buffer at $T \sim 700^{\circ} \mathrm{C}$. The experimental evidence is that the variation of $\sigma$ with $h$ does not depend on the inttal roughness. Indeed the slope $\mathrm{d} \sigma / \mathrm{d} h$ is found to be the same for different residual roughnesses measured by RHEED. Although we are limited in our experimental characterisation of surfaces, it is quite remarkable that the original surface state issued from growth and thermal annealing is not relevant in the subsequent development of kinetıc roughening. In figure 7 , a flattening of the curve $\sigma$ versus $h$ is clearly observed for the largest values of the film thickness. In this range of thicknesses, the RHEED patterns show the large size of the secondary difraction spots due to new crystallites and diffraction spots with lengths close to their widths. This apparent saturation of kinetic roughening is reproducible. Its interpretation on the strict basis of experimental results is rather complicated. As described in the previous section about experımental conditions, this flattenıng may only reveal a limit of the electron diffraction technique at grazing incidence in the analysis of rough surfaces.

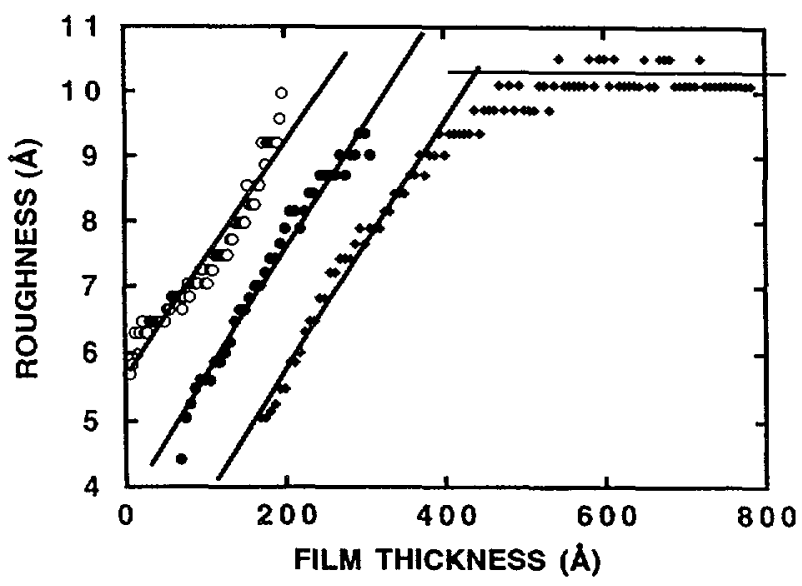

Fig. 7. - Roughness perpendicular to the surface deduced from the FWHM of diffraction Bragg peaks during the slicon deposition at $T=300^{\circ} \mathrm{C}$. This characterizes the effect of cycles of growth and annealing on the residual roughness and on the appearıng roughness during the growth (see text)

3.3 CREATION OF DEFECTS DURING EPITAXIAL GROWTH. - Figure 8 clearly shows that the diffraction pattern obtained after the growth of a thick silicon film in the temperature range $300^{\circ} \mathrm{C}-400{ }^{\circ} \mathrm{C}$, is very different from that given in figure 1 . The spotty diagram provides clear evidence for the deterioration of the epitaxial growth. The new Bragg spots seen on the pattern are a clear signature of well defined defects in the film. When the new spots appear, the absence of contınuous rings shows that randomly distributed silicon grains are not present. Indexation of the complete diffraction pattern along a [1 10$]$ azımuth can be done at this first 


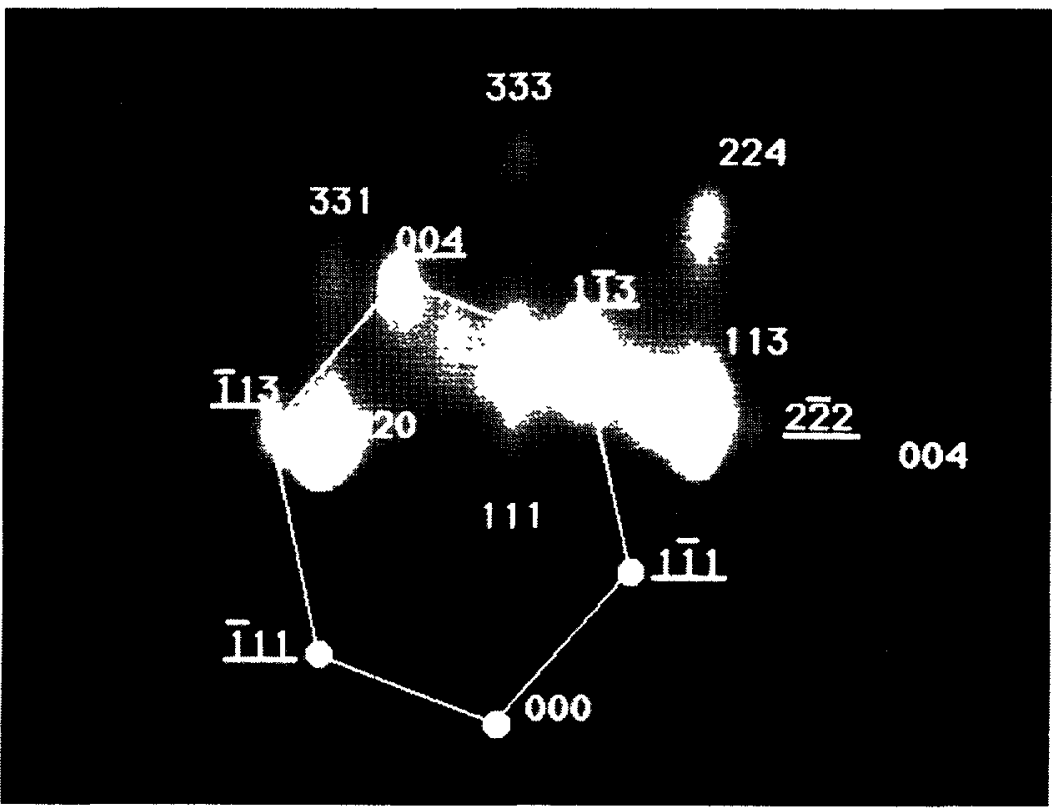

Fig. 8. - RHEED pattern after growth of a thick film at low temperatures. The electron beam is along the [1T0] azimuth. The complete indexation of extra spots clearly shows the appearance of a new type of crystal.

stage. This is what appears in figure 8 . The full diagram can be analysed by taking only two types of crystal into account. One diffraction pattern is associated with the original crystal exhibitıng a rough surface. The second one is consistent with the existence of new silicon crystals rotated by $180^{\circ}$ around the $\langle 112\rangle$ axis of the substrate. Both indices and angles in the patterns with beam along azimuth [1 $1 \overline{1} 0]$ support this analysis. However we cannot exclude that the broad Bragg peaks of the initial crystal do not hide the RHEED pattern of another type of appearing defects, especially the defects already reported [19] during the growth of silicon on $\mathrm{Si}(111)$ at low temperatures. These defects are thought to be twins which exhibit a $180^{\circ}$ rotation around the $\langle 111\rangle$ axis. In figure 8 , this would give a RHEED pattern deduced from the original one by a rotation of $180^{\circ}$ around the $\langle 111\rangle$ axis. Every Bragg peak introduced by these twinned crystals would be in the vicinity of a Bragg peak of the substrate. Therefore if such defects are present, then our measuremants are not able to reveal them unambiguously. At a later stage, further nucleations of crystals with other orientations appear. This corresponds to a new step toward a polycrystalline growth. Due to the apparent increasing number of these orientations, a quantıtatıve analysis of this new step in the breakdown of the epitaxial growth is difficult on the basis of RHEED patterns. In figure 9, we report the intensity of the 113 diffraction peak associated with the new crystal versus the film thickness (the location of this spot in the RHEED pattern is described in Fig. 8). The diffraction evidence for these crystals does not appear at the beginning of the growth on the flat surface but only after development of a significant roughness. Thereafter we observe a continuous increase of the diffraction intensity associated with these new crystals. At temperatures higher than $400{ }^{\circ} \mathrm{C}$, the diffraction evidence of this effect is hardly detectable even after the growth of rather thick films. This is also the temperature range where the effect of kinetic roughening is markedly decreased as shown in figure 5 . Therefore we observe a mechanism for the breakdown of the 


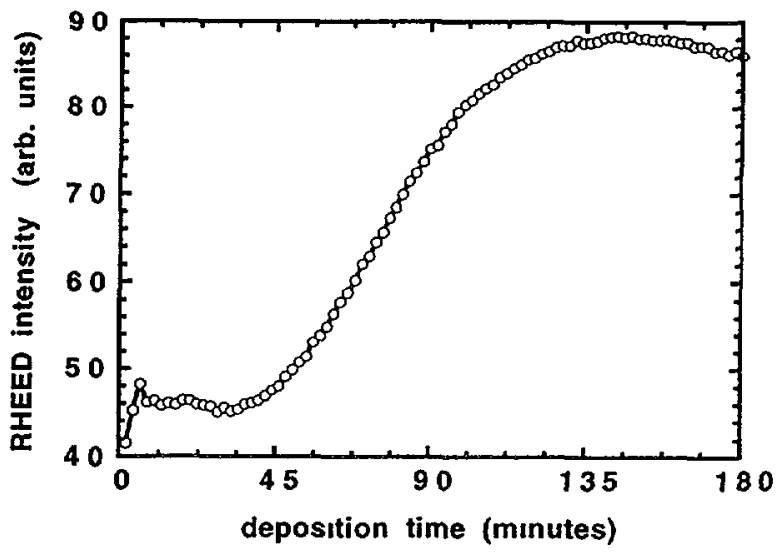

Fig. 9. - Measurement of the intensity of the appearing Bragg spot versus the deposition time.

epitaxial growth on (111) silicon surface different from the usual crystal-amorphous transition observed at lower temperatures and which appears to be induced by the kinetic roughening. This crystallographic identification of the initial nucleation of misoriented crystals during growth on (111) silicon surface also provides some further information on the increasing defect density reported in reference [19] during epitaxial growth on silicon (111) in the same range of temperatures.

\section{Conclusion.}

Although it is a general and characteristic behaviour of the surface morphology durıng epitaxial growth at low temperatures, the specific and in situ investigations of kinetic roughening during growth of thick films (several hundred angstroms) are not numerous. It is however a central problem of molecular beam epitaxy seen as a dynamical process. For semiconductors, it can leads to specific and spectacular effects like progressive disordering or amorphisation which to our knowledge have not received a satisfactory explanation although they have been repeatedly observed.

In this paper by means of analysis of RHEED patterns, we have experımentally observed the systematic occurrence of a roughness during the growth of sllicon at different temperatures. Within the limits of the RHEED technique in the analysis of the surface roughness, we have determined some characteristıcs of this roughness. Durıng the homoepitaxial growth of silicon on the Si(111) face, our experimental results indicate that the surface width vary linearly with the film thickness. This dependence of surface roughness with the film thickness is in marked difference with our previous results on the ron growth which have been obtained using exactly the same experimental method. This suggests that the details of mass transport mechanisms on the surface play an important role in the subsequent development of this roughness. Furthermore growth experıments performed at different temperatures indicate that the developping roughness is controlled by a thermally activated mechanism. This activation energy is $E_{\mathrm{d}} \sim 0.65 \mathrm{eV}$. The identification of the mechanısm responsible for this activation energy is beyond these experimental results. We have made an extrapolation of the temperature variation of the surface width to relevant temperatures for high quality silicon growth. This suggests that the occurrence of a kinetic roughness may be a relevant parameter in the determination of the usual experimental conditions for the MBE growth of silicon. 
After the occurrence of a significant amount of roughness, we have observed the spontaneous breakdown of the epitaxial growth. At very low temperatures, an amorphous phase readily follows the loss of the crystalline order. In medium range temperatures $\left(250^{\circ} \mathrm{C}\right.$ $400^{\circ} \mathrm{C}$ ), we have identified a progressive appearance of specific crystalline defects. Whatever the detailed mechanism, this epitaxial breakdown is a consequence of crystal growth by means of a process far from equilibrium. To our knowledge, despite the significant remarks of reference [14], a satisfactory description of silicon disordering durıng sllicon MBE growth is not established. It is then an aim of this paper to add some further experimental insights into the knowledge of this problem. We have found that as the temperature is raised, this effect seems to disappear progressively. RHEED studies of the surface morphology suggest that the occurrence of a kinetic roughness is a key point in the breakdown of epitaxial growth. Finally for two different systems, we have shown that systematic acquisition and quantitative analysis of RHEED patterns reveal some major effects appearing during the epitaxial growth of thick films at low temperatures. However many points have been left open due to the intrinsic limits of this technique. They certainly require further experimental investigations using different experimental methods. This is the case for the direct and in situ analysis of the crystalamorphous transition during silicon growth, for the detailed description of roughness development in these two different regimes of growth and certainly also for the study of defect nucleation during silicon growth.

\section{Acknowledgments.}

It is our great pleasure to thank Le Thanh Vinh, J. Y. Natolı, F. Arnaud D'Avitaya, D. E. Wolf and J. Villain for enlightening discussions. One of us (J. C.) has greatly benefited from the discussions with participants at the winter school of Les Houches organised by R. Jullien, D. E. Wolf, J. Kertez and P. Meakin. This work has been supported by the European Community through the Esprit Basic research Action Project no. 3026. Grants from the French Minıstère de la Recherche et de la Technologie and the PIRMAT-CNRS are also gratefully acknowledged (Contracts n. 88R0973 and 90S0249).

\section{References}

[1] «Silicon Molecular Beam Epitaxy », vol. 1 \& 2, E. Kasper and J. C. Bean Eds. (CRC Press, Inc. Boca Raton, FL, 1988).

[2] Eaglesham D. J., Gossmann H.-J and Cerullo M., Phys. Rev. lett. 65 (1990) 1227.

[3] See for example, the definition of the epitaxial temperature, $T_{\text {epi }}$, in Allen F. and Kasper E., p. 68 of Ref. [1].

[4] Kunkel R., Poelsema B., Verheij L. K. and Comsa G., Phys. Rev. Lett. 65 (1990) 733 ;

Poelsema B., Kunkel R., Nagel N., Becker A. F., Rosenfeld G., Verheij L. K. and Comsa G., Appl. Phys. A 53 (1991);

Bott M., Michely T. and Comsa G., Surf. Sci 272 (1992) 161.

[5] Chevrier J., Le Thanh Vinh, Buys R. and Derrien J., Europhys. lett. 16 (1991) 737.

[6] Aarts J. and Larsen P. K., « Reflection High Energy Electron Diffraction and Reflection Electron Imaging of Surfaces » P. K. Larsen and P. J. Dobson Eds. (Plenum, New York, 1988) p. 460.

[7] Sakamoto T., Kawai N. J., Nakawaga T., Ohta K. and Kojıma T., Appl. Phys. Lett. 47 (1985) 617.

[8] Wang S. C. and Ehrlich G., Phvs. Rev. Lett. 67 (1991) 2509;

Wang S. C. and Ehrlich G., Phys. Rev. Lett. 70 (1993) 41

Fink H. W. and Ehrlich G., Surf. Sci. 143 (1984) 125 ;

Wang S. C. and Tsong T. T., Surf. Sci. 121 (1982) 85.

[9] Mo Y. W., Kleıner J., Webb M. B. and Lagally M. G., Phys. Rev. Lett. 66 (1991) 1998. 
[10] For a theoretical review on kinetic roughening see «Solids far from equilibrium: growth, morphology and defects $\gg$ C. Godreche ed. (Cambridge University Press, 1990) and Proc. of «Surface disorderıng: growth, roughening and phase transitions» R. Jullien, J. Kertesz, P. Meakin, D. Wolf Eds. (Winter School of Physics at Les Houches, France, Nova Science, New York, 1993).

[11] Villain J., J. Phys. France 1 (1991) 19.

[12] Kardar M., Parisi G. and Zhang Y.-C., Phys. Rev. Lett. 56 (1986) 889.

[13] He Y.-L., Yang H.-N., Lu T.-M. and Wang G.-C., Phys. Rev. Lett. 69 (1992) 3770.

[14] Eaglesham and Gilmer G. J., to appear in Proc. of « Surface disordering : growth, roughening and phase transitions », R. Jullien, J. Kertesz, P. Meakin, D. Wolf Eds. (Winter School of Physics at Les Houches, France, Nova Science, New York, 1993).

[15] Tringides M. C. and Lagally M. G., « Reflection High Energy Electron Diffraction and Reflection Electron Imaging of Surfaces » P. K. Larsen and P. J. Dobson Eds. (NATO Series, Plenum, New York, 1988).

[16] S. Ino, «Reflection High Energy Electron Diffraction and Reflection Electron Imaging of Surfaces », P. K. Larsen and P. J. Dobson Eds. (Plenum New York, 1988) p. 3.

[17] It is possible to make an estımate of the error introduced by the convolution of both disorder and size effect in our analysis of surface roughness. If $\Delta G_{\perp}$ is the measured length of the Bragg peak perpendicular to the surface and $\Delta G_{\|}$, the measured length parallel to the surface, then a reasonable value for the intrinsic $\Delta G_{\mathrm{r}}$ associated with the roughness is (see Guinier A., «X Ray Diffraction $»(W$. H. Freeman and Company, San Francisco CAL 1963) p. 143):

$$
\Delta G_{\Lambda}=\sqrt{\Delta G_{\perp}^{2}-\Delta G_{\|}^{2}} .
$$

In the linear part of the curve $\sigma$ versus h, a usual experımental value for the ratio $\Delta G_{\perp} / \Delta G_{1}$ is about 3 or larger. This means that the difference between $\Delta G_{\perp}$ and $\Delta G_{\mathrm{r}}$ should be about $5 \%$ or less. This gives an indication of how approximate our method is. As the ratio $\Delta G_{\perp} / \Delta G_{1}$ becomes close to 2, with a difference between $\Delta G_{\perp}$ and $\Delta G_{\mathrm{r}}$ becoming larger than $10 \%$, one experimentally observes an apparent saturation in the increase of the surface roughness. As mentioned in the text, this saturation may indeed be the sign that the crystalline coherence is, at this stage, the important factor to determine $\Delta G_{\perp}$ with a limited effect of the surface width.

[18] Voigtländer B. and Zinner A., Surf. Sc. Lett. 292 (1993) L775.

[19] Weir B. E., Freer B. S., Headrick R. L., Eaglesham D. J., Gilmer G. H., Bevk J. and Feldman L. C., Appl Phys. Lett. 59 (1991) 204. 